\title{
A CHARACTERIZATION OF POSITIVE LINEAR FUNCTIONALS AND OSCILLATION CRITERIA FOR MATRIX DIFFERENTIAL EQUATIONS
}

\author{
TERRY WALTERS
}

\begin{abstract}
Etgen and Pawlowski have recently given criteria for oscillation of matrix differential equations which involve the value of positive linear functionals on the matrices appearing in a matrix differential equation. We characterize these functionals and indicate relationships to eigenvalue criteria for oscillation. Our results are also useful for the detection of the oscillation of particular matrix differential equations.
\end{abstract}

1. Introduction. In this article we examine positive linear functionals on the linear space of square matrices of order $n$. We show that there is a one-to-one correspondence between nonnegative definite matrices and positive linear functionals and we bound the value of the positive linear functional on a given matrix by the eigenvalues of the given matrix. These results are useful for the detection of the oscillation of matrix differential equations and we discuss this application in $\$ 4$ of this article.

2. Preliminaries. Let $M_{n}$ denote the linear space of all $n \times n$ real matrices over the reals. $A^{T}$ will denote the transpose of $A$.

Definition 2.1. A real linear functional is said to be positive if

(i) $g\left(A^{T} A\right) \geqslant 0$ for every $A \in M_{n}$;

(ii) $g\left(A^{T}\right)=g(A)$ for every $A \in M_{n}$.

The following well-known lemmas will be needed in the sequel. The proofs of these lemmas as well as the definitions of terms from linear algebra can be found in [1].

Lemma 2.2. For any $n \times n$ real matrix $A, A^{T} A$ is positive semidefinite. Conversely, if $B$ is positive semidefinite, then there exists an $n \times n$ matrix $A$ such that $B=A^{T} A$.

For $A, B \in M_{n}$ let $A \otimes B$ denote the coordinatewise product $\left[a_{i j} b_{i j}\right], i, j=$ $1,2, \ldots, n$.

Lemma 2.3. If $A, B \in M_{n}$ and $A, B$ are nonnegative definite, then $A \otimes B$ is also nonnegative definite.

In the sequel the norm of a vector will be the Euclidean norm. For $D \in M_{n}$, we

Presented to the Society, January 21, 1979; received by the editors May 30, 1979 and, in revised form, March 23, 1979.

AMS (MOS) subject classifications (1970). Primary 34C10.

Key words and phrases. Oscillation, matrix differential equations, positive linear functionals.

(C) 1980 American Mathematical Society 0002-9939/80/0000-0059/\$02.25 
let

$$
\|D\|=\sup _{\|v\|=1}\|D v\|
$$

where $v$ ranges over all column $n$-vectors.

3. Main results. The first result is a characterization of positive linear functionals on $M_{n}$. We let $\langle$,$\rangle denote the inner product on R^{n}$ and $u$ will denote the column vector in $R^{n}$ such that $u_{i}=1, i=1,2, \ldots, u$. Let $I$ denote the identity matrix.

TheOREM 3.1. Suppose that $g$ is a positive linear functional. Then there exists a nonnegative definite matrix $\left[\alpha_{i j}\right] \in M_{n}$ such that

$$
g(A)=\left\langle\left(\left[\alpha_{i j}\right] \otimes A\right) u, u\right\rangle,
$$

for every $A \in M_{n}$. Conversely, if $\left[\alpha_{i j}\right]$ is a nonnegative definite matrix, then the linear functional $g$, defined by (3.1) for $u$ as above, is a positive linear functional.

Proof. Suppose that $g$ is a positive linear functional. Let $C_{i j}, i, j=1,2, \ldots, n$, be the matrix in $M_{n}$ every entry of which is zero except the $i j$ th entry which is 1 . Then we obviously have

$$
A=\left[a_{i j}\right]=\sum_{i, j=1}^{n} a_{i j} C_{i j}
$$

and

$$
g(A)=\sum_{i, j=1}^{n} a_{i j} g\left(C_{i j}\right) .
$$

Now define $\alpha_{i j}=g\left(C_{i j}\right)$. Then we have $\alpha_{i j}=\alpha_{j i}$ from Definition 2.1(ii) and

$$
g(A)=\left\langle\left(\left[\alpha_{i j}\right] \otimes A\right) u, u\right\rangle \text {. }
$$

We shall prove that the matrix $\left[\alpha_{i j}\right]$ is nonnegative definite. To this end, assume that $\left[\alpha_{i j}\right]$ is not nonnegative definite. Then there exists $y \in R^{n}$ such that

$$
\left\langle\left[\alpha_{i j}\right] y, y\right\rangle=\sum_{i, j=1}^{n} \alpha_{i j} y_{i} y_{j}<0,
$$

where $\|y\|=1$. Now we construct a matrix $N=\left[n_{i j}\right]$ as follows: we form (by the Gram-Schmidt process) an orthonormal basis $\left\{y, x_{2}, x_{3}, \ldots, x_{n}\right\}$ of $R^{n}$ and let $N$ be the matrix whose columns are the vectors of this basis in the given order. Then it is easy to check that $N^{T}$ is the inverse of $N$. Let $B=N C_{11} N^{T}$. Then $B$ is nonnegative definite because its eigenvalues are $\lambda_{1}=1, \lambda_{i}=0$ for $i=2, \ldots, n$. Now we have

$$
\begin{aligned}
g(B) & =\left\langle\left(\left[\alpha_{i j}\right] \otimes B\right) u, u\right\rangle=\sum_{i, j=1}^{n} \alpha_{i j} b_{i j}=\sum_{i, j=1}^{n} \alpha_{i j}\left(\sum_{j=1}^{n} \lambda_{k} n_{i k} n_{j k}\right) \\
& =\sum_{i, j=1}^{n} \alpha_{i j} n_{i 1} n_{j 1}=\sum_{i, j=1}^{n} \alpha_{i j} y_{i} y_{j}<0 .
\end{aligned}
$$

This is a contradiction to the positiveness of $g$. Consequently, $\left[\alpha_{i j}\right]$ is nonnegative definite. The converse follows from Theorem 1, p. 94 of [1] and the equalities 


$$
g(B)=\sum_{i, j=1}^{n} \alpha_{i j} b_{i j}=\sum_{i, j=1}^{n} \alpha_{i j} b_{j i}=g\left(B^{T}\right) .
$$

The following theorem concerns itself with the fact that the norm of a positive linear functional is equal to its value at the identity matrix. This statement is in Corollary (4.5.3) of Rickart's book [6] but we give an independent proof since Rickart considers complex Banach algebras and his proof does not carry over to the case of a real Banach algebra.

TheOREM 3.2. Let $g, a$ positive linear functional, be given. Then if $g(B)=$ $\left\langle\left(\left[\alpha_{i j}\right] \otimes B\right) u, u\right\rangle$ and $\lambda_{1}, \ldots, \lambda_{n}$ are the eigenvalues of $\left[\alpha_{i j}\right]$ we have

$$
\|g\|=g(I)=\sum_{i=1}^{n} \lambda_{i}
$$

Proof. Let $D \in M_{n}$ be given with the property that $\|D\|=1$. Let $S$ and $S_{k}$ be the symmetric and skew-symmetric part of $D$ respectively. Then $D=S+S_{k}$ and

$$
\begin{aligned}
g(D) & =\left\langle\left(\left[\alpha_{i j}\right] \otimes D\right) u, u\right\rangle=\left\langle\left(\left[\alpha_{i j}\right] \otimes\left(S+S_{k}\right)\right) u, u\right\rangle \\
& =\left\langle\left(\left[\alpha_{i j}\right] \otimes S\right) u, u\right\rangle+\left\langle\left(\left[\alpha_{i j}\right] \otimes S_{k}\right) u, u\right\rangle \\
& =\left\langle\left(\left[\alpha_{i j}\right] \otimes S\right) u, u\right\rangle
\end{aligned}
$$

since $\alpha_{i j} s_{k i j}=-\alpha_{j i} s_{k i j}$, where $S_{k}=\left[s_{k i j}\right]$. Thus the skew-symmetric part of $D$ has no effect on $g(D)$. Now we show that all the eigenvalues of $S$ are bounded above in absolute value by 1 . Let $\mu$ be an eigenvalue of $S$ and $x_{\mu}$ a corresponding eigenvector with $\left\|x_{\mu}\right\|=1$. Then we have $S x_{\mu}=\mu x_{\mu}$ and

$$
\begin{aligned}
\left\langle S_{k}^{T} S x_{\mu}, x_{\mu}\right\rangle & =\left\langle\mu S_{k}^{T} x_{\mu}, x_{\mu}\right\rangle=0, \\
\left\langle S S_{k} x_{\mu}, x_{\mu}\right\rangle & =\left\langle S_{k} x_{\mu}, S x_{\mu}\right\rangle=\left\langle S_{k} x_{\mu}, \mu x_{\mu}\right\rangle=\mu\left\langle S_{k} x_{\mu}, x_{\mu}\right\rangle=0
\end{aligned}
$$

since $S_{k}$ is skew-symmetric. Let us notice now that the norm of $D$ equals the largest eigenvalue of $D^{T} D$ and that

$$
D^{T} D=\left(S+S_{k}\right)^{T}\left(S+S_{k}\right)=S^{2}+S_{k}^{T} S+S S_{k}+S_{k}^{T} S_{k}
$$

From (3.7) and (3.8) we obtain

$$
\begin{aligned}
\left\langle D^{T} D x_{\mu}, x_{\mu}\right\rangle & =\left\langle S^{2} x_{\mu}, x_{\mu}\right\rangle+\left\langle S_{k}^{T} S x_{\mu}, x_{\mu}\right\rangle+\left\langle S S_{k} x_{\mu}, x_{\mu}\right\rangle+\left\langle S_{k}^{T} S_{k} x_{\mu}, x_{\mu}\right\rangle \\
& =\left\langle S^{2} x_{\mu}, x_{\mu}\right\rangle+\left\langle S_{k}^{T} S_{k} x_{\mu}, x_{\mu}\right\rangle \geqslant\left\langle S^{2} x_{\mu}, x_{\mu}\right\rangle=\mu^{2} .
\end{aligned}
$$

This inequality along with the Courant-Fisher theorem gives

$$
\mu^{2}=\left\langle S^{2} x_{\mu}, x_{\mu}\right\rangle \leqslant 1 .
$$

Consequently, $|\mu| \leqslant 1$.

From the fact that all the eigenvalues of $S$ are bounded in absolute value by 1 , it follows that $I-S$ is nonnegative definite and, using Lemma 2.3, we have that $\left[\alpha_{i j}\right] \otimes(I-S)$ is nonnegative definite. For the particular vector $u$ we have

$$
\left\langle\left(\left[\alpha_{i j}\right] \otimes(I-S)\right) u, u\right\rangle \geqslant 0,
$$


from which we obtain

$$
g(D) \leqslant \sum_{i=1}^{n} \alpha_{i i}
$$

since the $\otimes$ product is easily seen to be distributive. From the well-known fact that the trace of a matrix is the sum of its eigenvalues we have

$$
\sum_{i=1}^{n} \alpha_{i i}=\sum_{i=1}^{n} \lambda_{i}=g(I)
$$

From (3.12) and (3.13) we have

$$
\|g\|=\sup _{\|D\|=1}|g(D)|=g(I),
$$

which completes the proof.

THEOREM 3.3. Let $g$ be a positive linear functional with norm 1. Then for every symmetric $D \in M_{n}$ we have

$$
\lambda_{m}(D) \leqslant g(D) \leqslant \lambda_{M}(D)
$$

where $\lambda_{m}(D)$ is the smallest eigenvalue of $D$ and $\lambda_{M}(D)$ is the largest eigenvalue of $D$.

Proof. Note first that $D-\lambda_{m}(D) I$ is nonnegative definite. From Lemma 2.4 we have that $\left[\alpha_{i j}\right] \otimes\left(D-\lambda_{m}(D) I\right)$ is nonnegative definite and, as in Theorem 3.2, we have

$$
\left\langle\left(\left[\alpha_{i j}\right] \otimes D\right) u, u\right\rangle \geqslant\left\langle\lambda_{m}(D)\left(\left[\alpha_{i j}\right] \otimes I\right) u, u\right\rangle .
$$

From Theorem 3.2 it follows that

$$
g(D) \geqslant \lambda_{m}(D) \sum_{i=1}^{n} \alpha_{i i}=\lambda_{m}(D) .
$$

A similar argument covers the assertion on the maximum eigenvalue $\lambda_{M}(D)$.

4. Applications. In [2] Etgen and Pawlowski showed that many of the current results in the oscillation theory of second order matrix differential equations are consequences of a theorem which we quote below. This theorem is concerned with oscillation of solutions to

$$
\left(P(x) Y^{\prime}\right)^{\prime}+Q(x) Y=0
$$

where $P(x)$ and $Q(x)$ are $n \times n$ symmetric matrices of functions of a real variable and a solution $Y(x)$ is defined to be an $n \times n$ matrix of functions of a real variable such that (4.1) is satisfied everywhere on $[a, \infty)$ for some $a>0$.

Definition 4.2. Equation (4.1) is said to be oscillatory if for every solution $Y(x)$ satisfying

$$
Y^{T}(x) P(x) Y^{\prime}(x)=Y^{T \prime}(x) P(x) Y(x)
$$

the determinant of $Y(x)$ has an unbounded set of zeroes on $[a,+\infty)$.

Theorem A (Etgen And Pawlowski). Let $P(t)$ be positive definite. If there exists a positive linear functional such that the scalar equation

$$
\left[g(P(x)) Y^{\prime}\right]^{\prime}+g(Q(x)) Y=0
$$

is oscillatory, then (4.1) is oscillatory. 
Using Theorem $\mathrm{A}$ and Theorem 3.1 we give the following example. This example is of particular interest since the off-diagonal elements of $Q$ are causing oscillation and many previously known matrix oscillation criteria fail. In particular, the eigenvalues of $\int_{0}^{t} Q(s) d s$ are all bounded.

EXAMPLE 4.3. Let

$$
\begin{aligned}
P(t)= & {\left[\begin{array}{ccc}
1 & -1 / t & 0 \\
-1 / t & 1 / t & 0 \\
0 & 0 & 1 / t
\end{array}\right], Q(t)=\left[\begin{array}{ccc}
0 & e^{-t} & -1 / t^{2} \\
e^{-t} & 0 & 0 \\
-1 / t^{2} & 0 & 0
\end{array}\right], } \\
{\left[\alpha_{i j}\right] } & =\left[\begin{array}{ccc}
1 / 3 & 0 & -1 / 6 \\
0 & 1 / 3 & 0 \\
-1 / 6 & 0 & 1 / 3
\end{array}\right] .
\end{aligned}
$$

Then it is easy to see that $g(Q(t))=1 / 3 t^{2}$ and $g(P(t))=1 / 3+2 / 3 t$. To show that the resulting scalar equation is oscillatory, we note that eventually $2>(1+$ $2 / t$ ) and employ the Sturm-Picone theorem [5] to reduce the question of oscillation to the comparison equation $u^{\prime \prime}+1 / 2 t^{2} u=0$. This equation is oscillatory by Theorem 2.1 of [8].

Noticing that from the form of the scalar comparison equation in Theorem A we can assume that $g$ has norm one, Theorem 3.3 suggests that some eigenvalue criterion might be stronger than the criteria of Theorem A using positive linear functionals. In fact, Tomastik [9] and [10] has some results in this direction. It should be noted however, that St. Mary has shown in [7] that the oscillation of $\left(\lambda_{m}\left(P(x) y^{\prime}\right)\right)^{\prime}+\lambda_{M}(Q(x)) y=0$ is not sufficient for the oscillation of (4.1).

\section{REFERENCES}

1. R. Bellman, Introduction to matrix analysis, McGraw-Hill, New York, 1960.

2. G. J. Etgen and J. F. Pawlowski, A comparison theorem and oscillation criteria for second order differential systems, Pacific J. Math. 72 (1977), 59-69.

3. A. G. Kartsatos, Oscillation of nonlinear systems of matrix differential equations, Proc. Amer. Math. Soc. 30 (1971), 97-101.

4. A. G. Kartsatos and T. J. Walters, Origins of oscillation criteria of operator differential equations in Hilbert space, J. Math. Anal. Appl. 67 (1979), 12-16.

5. K. Kreith, Oscillation theory, Lecture Notes in Math., vol. 324, Springer-Verlag, Berlin and New York, 1973.

6. C. E. Rickart, Banach algebras, Van Nostrand, New York, 1960.

7. D. F. St. Mary, On transformation and oscillation of linear differential systems, Canad. J. Math. 19 (1977), 392-399.

8. C. A. Swanson, Comparison and oscillation theory of linear differential equations, Academic Press, New York, 1968.

9. E. C. Tomastik, Oscillation of nonlinear matrix differential equations of second order, Proc. Amer. Math. Soc. 19 (1968), 1427-1431.

10. (1971), 436-442.

, Oscillation of systems of second order differential equations, J. Differential Equations 9

Department of Mathematics, Pan American University, Edinburg, Texas 78539 